\title{
The fate of unattended stimuli and emotional habituation: Behavioral interference and cortical changes
}

\author{
Maurizio Codispoti ${ }^{1} \cdot$ Andrea De Cesarei $^{1} \cdot$ Simone Biondi $^{1} \cdot$ Vera Ferrari $^{2}$
}

Published online: 24 August 2016

(C) Psychonomic Society, Inc. 2016

\begin{abstract}
The interference produced by the viewing of emotional distractors has been interpreted as evidence that emotional cues are processed in a fairly mandatory fashion, and that they divert attention from the primary ongoing task. However, few studies have examined how behavioral emotional interference varies with repeated presentation of the same emotional distractors. In two experiments, while participants were engaged in a parity judgment task, we investigated the effects of repetition of task-irrelevant emotional pictures, as reflected in both behavioral interference (Experiments 1 and 2) and neural activity (Experiment 2). Both experiments showed that the slowing of reaction times that was observed when viewing emotional, compared to neutral, scenes disappeared after only a few repetitions, suggesting diminished attention allocation to repeated emotional pictures. Conversely, in Experiment 2, neural correlates of picture processing revealed that the late positive potential (LPP) amplitude continued to be enhanced for emotional, compared to neutral, distractors despite picture repetition and the presence of a concurrent task. Altogether, these findings suggest that while evaluative processes are mandatory, and continue to engage cortico-limbic appetitive and defensive systems even after massive repetition, as suggested by the affective modulation of the LPP, attentional processes are not necessary after
\end{abstract}

Electronic supplementary material The online version of this article (doi:10.3758/s13415-016-0453-0) contains supplementary material, which is available to authorized users.

Maurizio Codispoti

maurizio.codispoti@unibo.it

1 Department of Psychology, University of Bologna, Bologna, Italy

2 Department of Neuroscience, University of Parma, Parma, Italy several repetitions of the same stimulus, as indicated by the rapid decline of behavioral emotional interference.

Keywords Emotion $\cdot$ Attention $\cdot$ Repetition $\cdot$ ERPs · Response times

Survival depends on the ability to evaluate the emotional significance of stimuli, and adapt one's behavior accordingly. When someone encounters an emotional stimulus this sets off a cascade of brain responses. Several studies suggest that emotional cues activate cortico-limbic appetitive and defensive systems, which, in turn, enhance attention allocation, and prepare the organism for action (Anderson, Laurent, \& Yantis, 2011; Gottlieb, 2012; Hickey, Chelazzi, \& Theeuwes, 2010; Lang, Bradley, \& Cuthbert, 1997; Lang \& Bradley, 2010; LeDoux, 2012; Pourtois, Schettino, \& Vuilleumier, 2013; Raymond, \& O'Brien, 2009). While in most circumstances the activation of the motivational systems is associated with enhanced attention, these two processes may be differentially affected by contextual factors (e.g., stimulus repetition). In the present study, we aimed to examine the relationships between the engagement of motivational systems and attention during the processing of emotional stimuli.

Research has shown that emotional stimuli engage attentional resources, and hence disrupt performance (i.e., elongate reaction time) in concurrent tasks. Interference effects have been reported using a variety of visual and acoustic tasks, suggesting that, compared to neutral stimuli, pictures depicting both pleasant and unpleasant contents draw more on attentional resources, leaving them less available for task processing (Bradley, Cuthbert, \& Lang, 1999; Calvo, Gutiérrez-García, \& Del Líbano, 2015; De Cesarei \& Codispoti, 2008; Ferrari, Mastria, \& Bruno, 2014; Hartikainen, Ogawa, \& Knight, 2000; Ihssen, 
Heim, \& Keil, 2007; Mitchell, Richell, Leonard, \& Blair, 2006; Most, Smith, Cooter, Levy, \& Zald, 2007; Padmala \& Pessoa, 2014; Schimmack, 2005). The interference produced by emotional distractors has been interpreted as evidence that the processing of emotional stimuli is not only prioritized, but that it occurs in a fairly mandatory fashion (Vuilleumier, Armony, Driver, \& Dolan, 2001). However, it is less clear how emotional interference is affected by stimulus repetition. A previous study which addressed this issue clearly indicates that although emotional words initially attract attention, after only a few repetitions they become less distracting in a performance task ("digit-parity task"), and act more like neutral stimuli (Harris \& Pashler, 2004). Therefore, the authors conclude that "this effect proved fleeting, apparently reflecting a momentary response of surprise (or something akin to surprise) that habituates very rapidly", and suggest that emotional stimuli are not so special after all, in terms of attracting attention (Harris \& Pashler, 2004).

Recently, researchers have begun to incorporate eventrelated potentials (ERPs) into the study of attentional capture by emotional stimuli. In terms of ERPs, it is well established that emotional pictures (pleasant and unpleasant) elicit a larger late positive potential (LPP) than neutral ones (Cuthbert, Schupp, Bradley, Birbaumer, \& Lang, 2000; Johnston, Miller, \& Burleson, 1986; Schupp, Flaisch, Stockburger, \& Junghöfer, 2006), and this cortical modulatory effect has been interpreted as reflecting both the engagement of attentional resources by emotional stimuli and the activation of motivational systems (Ferrari, Bradley, Codispoti, \& Lang, 2011; Lang, Bradley, \& Cuthbert, 1997; Schupp et al., 2006; Weinberg \& Hajcak, 2011).

Concerning the effects of stimulus repetition on the affective modulation of the LPP, a recent series of studies found that, even after massive repetition, emotional pictures continue to elicit a larger late positive potential than neutral ones (Codispoti, Ferrari, \& Bradley, 2006, 2007; Ferrari et al., 2011). On one hand, these LPP findings appear to be in contrast with the rapid decline of the emotional interference effect reported in previous behavioral studies (Harris \& Pashler, 2004); however, it should be noted that these studies used words as emotional distractors, which have been proved to be weaker stimuli, in terms of emotional engagement, when compared to pictures of natural scenes (Bayer, Sommer, \& Schacht, 2011; Bayer \& Schacht, 2014; Bradley, Mogg, Falla, \& Hamilton, 1998; De Houwer \& Hermans, 1994; Harris \& Pashler, 2004; Hinojosa, Carretié, Valcárcel, MéndezBértolo, \& Pozo, 2009). On the other hand, previous ERP studies examined the effects of stimulus repetition on the LPP affective modulation in free viewing contexts, in which emotional stimuli were attended (Codispoti, Ferrari \& Bradley, 2006, 2007). Less is known regarding the effects of repeated emotional scenes while participants are actively engaged in an unrelated task, which limits the amount of attentional resources that can be committed to the evaluation of affective, task-irrelevant stimuli and calls for competition between motivationally salient distractors and task relevant stimuli. This competition is expected to affect all processes which require attentional resources, and therefore can be used as a test to determine whether behavioral interference and the LPP are similarly related to attentional processes, or rather if they reflect different facets of emotional processing.

Our goal in the present study was to examine the effects of stimulus repetition on the processing of taskirrelevant emotional pictures, as reflected in both behavioral interference (Experiments 1 and 2) and neural activity (ERPs, Experiment 2) while participants were actively engaged in a parity judgment task, with pictures of natural scenes as distractors. In order to clarify the effect of repetition on the processing of emotional distractors, we presented a novel phase after the habituation phase where a new set of stimuli was presented. A reduction of emotional interference across blocks could be due to several factors such as a specific habituation to repeated stimuli, or it is possible that, along with successive presentations of task-irrelevant emotional items, processing of interfering information is inhibited in order to maximize task efficiency (Kelley \& Yantis, 2009). While both these possibilities predict that emotional interference should decrease following repeated presentations of the same stimuli, the introduction of a novel phase, where new pictures are presented, allows us to differentiate attentional capture to a specific stimulus (Harris \& Pashler, 2004), from a more general habituation to task-irrelevant stimuli (Kelley \& Yantis, 2009; Turatto \& Pascucci, 2016). The novel phase closely resembles the habituation blocks except for the specific picture set, which consists of new exemplars drawn from the same emotional categories. If behavioral interference results from attentional capture to an unexpected/unfamiliar item, which vanishes when a repeated stimulus becomes familiar, then a renewed interference effect should be observed when new images are presented in the novel phase. Alternatively, if repetition leads to a reduction in emotional processing of taskirrelevant information, the pattern during the novel phase should not differentiate from the immediately preceding trials, in spite of the change in stimulus.

\section{Experiment 1}

In Experiment 1, we examined the extent to which stimulus repetition affected the behavioral interference of task-irrelevant emotional pictures while participants were engaged in a parity judgment task. More specifically, participants rapidly judged whether two briefly 
presented digits were of the same parity (both odd or both even) or of a different parity ('yes' when they were both even or odd, 'no' if one was a mismatch; see Harris \& Pashler, 2004, and Wolford \& Morrison, 1980) while task-irrelevant pictures depicting pleasant, neutral or unpleasant contents appeared between the two digits on each trial. Figure 1 depicts the sequence of events in the present study. Pictures were repeated nine times each across three blocks in the habituation phase, then followed by a novel phase in which a new set of pictures, belonging to the same emotional categories, was presented.

\section{Method}

Participants A total of 31 healthy students (18 females) volunteered to participate in the experiment and signed an informed consent form. Mean age was 22.9 years $(\mathrm{SD}=$ 2.1), and all participants were right-handed.

Materials A total of 60 pictures, depicting erotic couples $(\mathrm{n}=20)$, neutral people $(\mathrm{n}=20)$ and mutilated bodies $(\mathrm{n}=20)$, were selected from the International Affective Picture System (IAPS; Lang, Bradley, \& Cuthbert, $2008)^{1}$, as well as from pictures available on the Internet. A total of 20 additional pictures, depicting neutral objects, were selected to be used only in the practice phase. Stimuli were presented on a 16 -in. monitor at $1,024 \times 768$ resolution and a refresh rate of $75 \mathrm{~Hz}$, controlled by an IBM computer. Each picture, subtending $9.15^{\circ}$ horizontal by 6.72 vertical degrees of visual angle, was presented foveally on a white background and was flanked by two numbers, each one subtending $0.42^{\circ}$ horizontal by 0.69 vertical degrees of visual angle. The distance between the edges of the image and the digits was $0.76^{\circ}$ of visual angle. Stimulus presentation and data collection were performed using E-Prime software (Schneider, Eschman, \& Zuccolotto, 2002).

Procedure As illustrated in Fig. 1, the experiment consisted of three habituation blocks followed by one novel block, each block consisting of 45 trials. During each trial, a fixation cross was presented in the center of the screen for $500 \mathrm{~ms}$. Subsequently, a picture flanked by

\footnotetext{
${ }^{1}$ IAPS codes were: Pleasant: 4669, 4670, 4659, 4658, 4680, 4659, 4664, 4690, 4810, 4611, 4651, 4800. Neutral: 2190, 2372, 2435, 2515, 2200, 2495, 2595, 2210, 2850, 4605, 7550, 2214, 2320. Unpleasant: 3000, $3010,3015,3102,3101,3051,3063,3100,3120,3170,3053,3064$, $3130,3060,3140,3266$. These IAPS pictures were complemented by other pictures of similar affective content and scene composition. Arousal ratings for IAPS pictures were: Pleasant, $\mathrm{M}=6.47(\mathrm{SD}=.38$ ); Neutral, $\mathrm{M}=3.46, \mathrm{SD}=.50$; Unpleasant, $\mathrm{M}=6.60, \mathrm{SD}=.54$. Valence ratings for IAPS pictures were Pleasant, $\mathrm{M}=6.64, \mathrm{SD}=.34 ; \mathrm{Neutral}, \mathrm{M}=$ $5.29, \mathrm{SD}=.53$; Unpleasant, $\mathrm{M}=1.62, \mathrm{SD}=.24$.
}

two numbers was presented for $150 \mathrm{~ms}$. Then, a blank screen was presented for $3 \mathrm{~s}$, during which behavioral responses to the parity judgment task were collected. Participants were instructed to focus on the two numbers while ignoring the picture, and to indicate whether they had the same parity (either both odds or both even) or not (one odd and one even or vice versa) by pressing one of two buttons. Participants were asked to respond as quickly and as accurately as possible.

In order to let participants become familiarized with the task, the experimental session started with a practice block of 20 trials presenting pictures of neutral objects flanked by two numbers. Data from the practice block were not reported. Four different sets of 15 pictures each (five pictures for each affective content) were created to increase the generalizability of the results beyond the specific features of a limited set of experimental stimuli. Each subject saw one set of pictures that was repeated 9 times throughout the habituation phase, three times in each block of 45 trials. After the habituation phase, a novel, previously unseen set of 15 pictures was presented (novel phase), and again repeated three times. Sets of pictures were counterbalanced across subjects so that, across participants, each set of pictures was seen equally often in the habituation or novel phase. Presentation order was pseudorandomized with the restriction that the same picture or a picture of the same valence could not occur in more than two consecutive trials.

Data collection and analysis Response times (RTs) and accuracy were collected. Analysis of RTs was performed only on correct responses. For each participant, RTs above or below two standard deviations from the mean were discarded. A repeated measures analysis of variance (ANOVA) with factors Valence (pleasant, neutral, unpleasant) and Block (four levels) was performed. In order to deal with violations of sphericity, a Huynh-Feldt correction was applied to the degrees of freedom. For each ANOVA test, we calculated and report the partial eta squared $\left(\eta_{p}^{2}\right)$, which reflects the proportion of the variance that is explained by experimental manipulations, over the total variance.

\section{Results and discussion}

Response times (RTs) As illustrated in Fig. 2, slower RTs were observed for emotional compared to neutral distractors during the first block. This emotional interference effect waned with picture repetition in blocks 2 and 3 . When new pictures were presented in the novel phase, a renewed interference of emotional content was observed. Analysis of RTs yielded a significant effect of Valence, $F(2,60)=9.11$, $\mathrm{p}<.001, \eta_{\mathrm{p}}^{2}=.233$, indicating slower RTs for emotional 
A

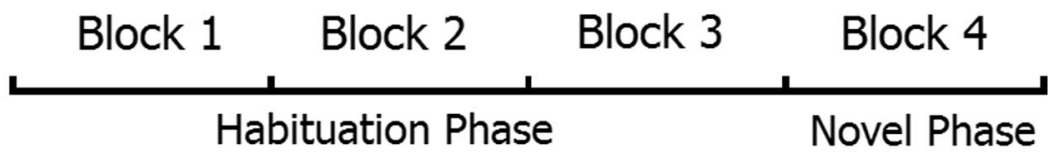

B

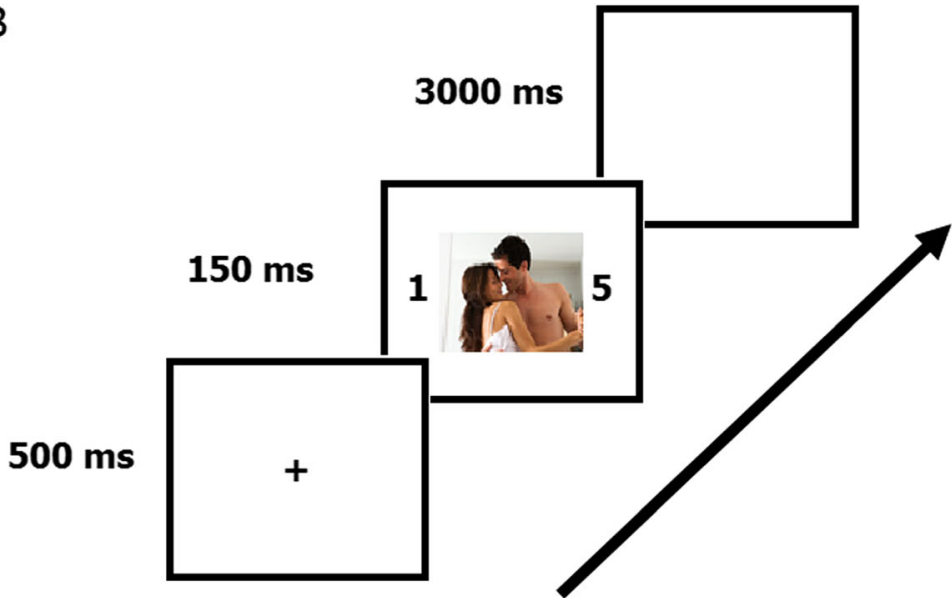

Fig. 1 (A) Schematic diagram showing the sequence of events in Experiment 1. (B) A sample trial from the task: A fixation crossdirected participants attention. Next, a picture flanked by two numbers was presented for $150 \mathrm{~ms}$. Then, a blank screen was presented for $3 \mathrm{~s}$, during which behavioral responses to the parity judgment task were

(pleasant and unpleasant) compared to neutral scenes, $\mathrm{F}_{\mathrm{s}}(1,30)$ $>12.62, \mathrm{p}_{\mathrm{s}}<.001, \eta_{\mathrm{ps}}^{2}>.296$, and no difference between pleasant and unpleasant stimuli, $\mathrm{F}(1,30)<1, \mathrm{p}=.380$. A significant main effect of Block was also found, $\mathrm{F}(3,90)=13.22, \mathrm{p}<.001, \eta_{\mathrm{p}}^{2}=.306$, indicating slower responses in block 1 compared to all other blocks, $\mathrm{F}_{\mathrm{s}}(1,30)>10.32, \mathrm{p}_{\mathrm{s}}<.01, \eta_{\mathrm{ps}}^{2}>.256$, and in block 2 compared

\section{EXPERIMENT 1}

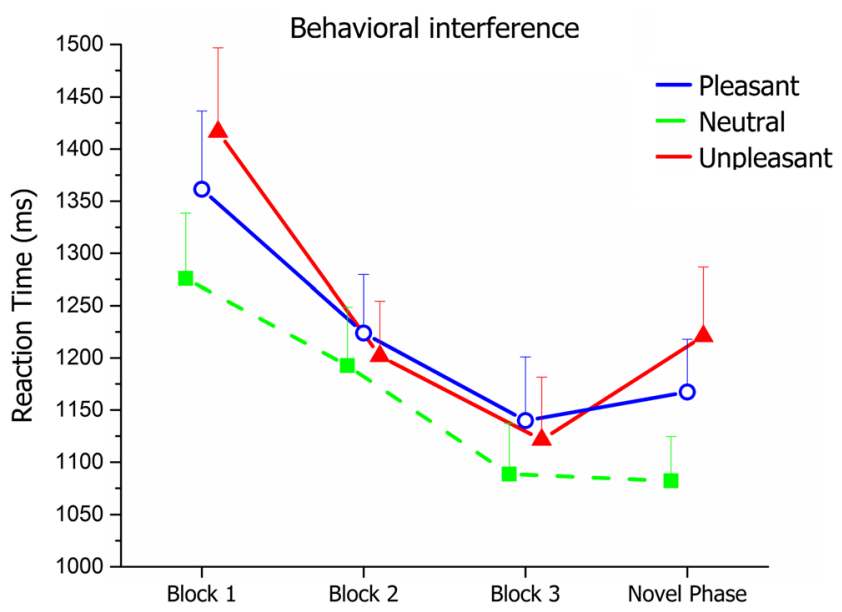

Fig. 2 Response times in the parity judgment task across the three habituation blocks, and one block containing novel pictures, plotted by distractor valence. Error bars represent one standard error of the mean collected. Participants were instructed to focus on the two numbers while ignoring the picture, and to indicate whether they had the same parity (either both odds or both even) or not (one odd and one even or vice versa) by pressing one of two buttons

to block $3, \mathrm{~F}(1,30)=5.50, \mathrm{p}=.026, \eta_{\mathrm{p}}^{2}=.155$, while block 3 did not differ significantly from the novel phase, $\mathrm{F}$ (1, $30)=2.79, \mathrm{p}=.105$. Importantly, a significant interaction Block $\times$ Valence was observed, $\mathrm{F}(6,180)=2.53, \mathrm{p}=.049$, $\eta_{p}^{2}=.078$. Following up on this interaction, the Valence effect was tested in each block: a significant affective modulation of RTs was observed in block $1, \mathrm{~F}(2,60)=5.45, \mathrm{p}=.014$, $\eta_{\mathrm{p}}^{2}=.154$, indicating slower responses to pleasant and unpleasant, compared to neutral stimuli, $\mathrm{F}_{\mathrm{s}}(1,30)>5.68, \mathrm{p}_{\mathrm{s}}<.05$, $\eta_{\mathrm{ps}}^{2}>.159$, but not in block 2 and 3 of the habituation phase, $\operatorname{Fs}(2,60)<1.35$, ps $>.267$. Crucially, the novel phase showed a significant affective modulation of RTs, $\mathrm{F}(2,60)=8.72$, $\mathrm{p}<.001, \eta_{\mathrm{p}}^{2}=.225$, with again slower responses to emotional, compared to neutral, stimuli, $\mathrm{F}_{\mathrm{s}}(1,30)>13.41, \mathrm{p}_{\mathrm{s}}<.001$, $\eta_{\text {ps }}^{2}>.309$, and no difference between pleasant and unpleasant pictures, $\mathrm{F}(1,30)=2.03, \mathrm{p}=.164$. Also, we calculated the difference scores (emotional minus neutral stimuli) in order to compare the emotional interference effect in block 1 and the novel phase, and we found that the two blocks showed similar emotional effects, $\mathrm{F}(2,60)<1, \mathrm{p}>.05$.

Accuracy Accuracy for each condition is shown in Table 1. Analysis of accuracy yielded a significant effect of Valence, $\mathrm{F}(2,60)=10.09, \mathrm{p}<.001, \eta_{\mathrm{p}}^{2}=.252$, indicating lower accuracy during viewing of pleasant and unpleasant stimuli, compared to neutral ones, $\mathrm{F}_{\mathrm{s}}(1,30)>17.35, \mathrm{p}_{\mathrm{s}}<.001, \eta_{\mathrm{ps}}^{2}>.366$, 
Table 1 Mean percentage correct and standard error (in parentheses) in the digit parity task for each picture content, block, and experiment

\begin{tabular}{|c|c|c|c|c|c|}
\hline & & Habituation & & & Novel phase \\
\hline \multirow[t]{4}{*}{ Experiment 1} & & Block 1 & Block 2 & Block 3 & Block 4 \\
\hline & Pleasant & $75.0(2.9)$ & $86.4(1.9)$ & $88.3(1.8)$ & 86.9 (1.6) \\
\hline & Neutral & $82.7(1.9)$ & $88.7(1.5)$ & $92.8(1.1)$ & $90.1(1.3)$ \\
\hline & Unpleasant & $75.0(3.0)$ & $86.5(1.8)$ & $88.2(1.9)$ & $87.9(1.9)$ \\
\hline \multirow[t]{3}{*}{ Experiment 2} & Pleasant & $86.3(2.7)$ & $91.9(1.9)$ & $94.6(1.4)$ & $91.8(2.6)$ \\
\hline & Neutral & $91.7(3.0)$ & $94.1(2.3)$ & $93.6(2.0)$ & $93.1(2.9)$ \\
\hline & Unpleasant & $89.4(3.4)$ & $90.7(3.0)$ & $93.9(1.8)$ & $89.8(3.6)$ \\
\hline
\end{tabular}

with no difference between pleasant and unpleasant pictures, $\mathrm{F}(1,30)=.06, \mathrm{p}=.814$. A significant Block effect was observed, $\mathrm{F}(3,90)=28.5, \mathrm{p}<.001, \eta_{\mathrm{p}}^{2}=.487$, indicating lower accuracy in block 1 compared to all the other blocks, $\mathrm{F}_{\mathrm{s}}(1,30)>31.94, \mathrm{p}_{\mathrm{s}}<.001, \eta_{\mathrm{ps}}^{2}>.516$, and in block 2 compared to block $3, F(1,30)=6.23, p=.018, \eta_{p}^{2}>.172$. The interaction between Block and Valence was not significant, $\mathrm{F}(6,180)=1.10, \mathrm{p}=.362$.

Experiment 1 showed that the emotional interference effect, here indexed by reaction time modulation, of taskirrelevant pictures declined with stimulus repetition. Emotional pictures drew more on attentional resources compared to neutral ones, leaving these resources less available for task processing, as highlighted by a reaction time slowdown in block 1, while this interference effect waned with increasing picture repetition (blocks 2 and 3). One hypothesis was that the decline of emotional interference across blocks reflected a general inhibition process of task-irrelevant stimuli. If this were the case, no interference effect of new emotional pictures would have been observed in the novel phase. In contrast, we found that the emotional effect recovered completely when novel pictures were presented, indicating that the decline of emotional interference across repetitions was related to the specific picture exemplars presented during the repetition blocks, rather than being a general inhibition of taskirrelevant emotional stimuli.

\section{Experiment 2}

Does emotion continue to modulate the processing of taskirrelevant pictures across several repetitions? The observation that emotional interference dissipates with repeated picture presentations suggests that the emotional content no longer impacts picture processing, supporting a scenario in which emotional distractors were filtered out. However, the filtering of task-irrelevant stimuli may occur at different stages of processing, and may involve different mechanisms. Recently, it has been shown that the mechanisms subserving attentional orienting are subject to profound and enduring plastic changes based on previous experience (Kelley \& Yantis, 2009; Qu,
Hillyard, \& Ding, 2016), and that habituation can selectively impact attentional processes (Turatto \& Pascucci, 2016). Therefore, the perceptual and semantic processing of taskirrelevant stimuli might occur independently of attentive processes (Cowan; 1988; Elliott \& Cowan; 2001; Gronau, Cohen, \& Ben-Shakhar, 2009; Öhman, 1992). If this is the case, then it should be possible to observe an emotional processing of the task irrelevant stimuli, which persists even when distractors are efficiently prevented from interfering with the ongoing primary task. To this end, in Experiment 2 we reproduced the paradigm used in Experiment 1, and used ERPs to track picture processing prior to overt response. We focused our analysis on the LPP, which has been suggested to reflect emotional processing of the semantic, rather than perceptual, properties of the stimuli (Bradley, Hamby, Löw, \& Lang, 2007; Codispoti, Mazzetti, \& Bradley, 2009; Codispoti, De Cesarei \& Ferrari, 2012; De Cesarei \& Codispoti, 2006; 2011). Moreover, the results of Experiment 1 seem to be in contrast with recent ERP findings on emotional habituation, which show that the emotional modulation of the LPP persists even when the same pictures are repeated 90 times (Codispoti et al., 2007). One possibility is that the LPP and behavioral interference reflect different facets of emotional processing, and are therefore differently affected by stimulus repetition. However, this interpretation is limited by the fact that, in previous ERP studies, emotional pictures were presented in a free viewing context, while emotional pictures in Experiment 1 were distractors that had to be ignored. To clarify these issues, in Experiment 2 we used the same paradigm as in Experiment 1, and we examined behavioral and cortical measures of affective processing.

\section{Method}

Participants A total of 15 healthy students (seven females) volunteered to participate in the experiment and signed an informed consent form. Mean age was 24.9 years $(\mathrm{SD}=$ 2.6), and all participants were right-handed.

Materials Picture stimuli were identical to those used in Experiment 1. Additionally, 90 new neutral images (objects 
and neutral animals) were selected to be presented during the practice block. The equipment used to present stimuli was the same as in Experiment 1.

Procedure Similar to Experiment 1, Experiment 2 consisted of a practice phase ( 90 trials), which was followed by three blocks for habituation and one block containing novel stimuli, each block consisting of 105 trials. In order to achieve a better signalto-noise ratio, the number of trials in each phase was augmented compared to Experiment 1; this also allowed us to investigate the interaction of emotion and repetition when the task is highly practiced (Harris \& Pashler, 2004). Throughout the three habituation blocks, a set of 15 pictures (five pleasant, five neutral, and five unpleasant) were repeatedly presented for a total of 21 times. In the novel phase, a new set of 15 pictures was presented and repeated seven times. As in Experiment 1, no more than two pictures depicting the same emotional content were presented subsequently, and repetitions occurred with the constraint that at least one image was interposed between two presentations of the same picture. Across subjects, each picture was assigned both to the habituation and to the novel phase.

EEG recording and processing Electroencephalogram (EEG) was recorded at a sampling rate of $500 \mathrm{~Hz}$ and a resolution of $0.12 \mu \mathrm{V}$ using a 59 channel Electro-Cap connected to a SA Instrument CO (San Diego, CA, USA) UF-64/72BA amplifier and in-house developed software. Impedance of each sensor was kept below $10 \mathrm{k} \Omega$. Eye movements were recorded at a sampling rate of $500 \mathrm{~Hz}$ and a resolution of $0.24 \mu \mathrm{V}$ from two bipolar couples of electrodes, placed respectively $1 \mathrm{~cm}$ above and below the right eye and $1 \mathrm{~cm}$ left and right to the side of the eyes. Both EEG and ocular signal were on-line filtered from 0.01 to $100 \mathrm{~Hz}$. Off-line analysis was performed using Emegs (Peyk, De Cesarei, \& Junghöfer, 2011). First, eye movements were subtracted from the EEG on a trial by trial basis, based on the data from the monopolar horizontal and vertical EOG, and using a regressive procedure (Gratton, Coles, \& Donchin, 1983). Then, data were low-pass filtered at $30 \mathrm{~Hz}$. Trials and sensors containing artifacts were detected through a statistical procedure (Junghöfer, Elbert, Tucker, \& Rockstroh, 2000). In each trial, if a high number of neighboring bad sensors was present, then the whole trial was discarded; for the remaining trials, sensors containing artifacts were replaced by interpolating the nearest good sensors. The percentage of good trials was $85 \%$; this percentage did not change across blocks or picture content. Finally, data were rereferenced to the average of all channels. A baseline correction based on the $100 \mathrm{~ms}$ prior to stimulus onset was performed. Processed data were averaged according to factors Valence and Block. The LPP was scored as the average of the ERP waveform in the 400-800 ms time interval after stimulus onset at centroparietal sensor group ( $\mathrm{FC} 1, \mathrm{FCz}, \mathrm{FC} 2, \mathrm{C} 3, \mathrm{C} 1, \mathrm{Cz}, \mathrm{C} 2, \mathrm{C} 4, \mathrm{CP} 3$, $\mathrm{CP} 1, \mathrm{CPz}, \mathrm{CP} 2, \mathrm{CP} 4, \mathrm{P} 3, \mathrm{P} 1, \mathrm{Pz}, \mathrm{P} 2$, P4; see figure showing the sensor positions in the Supplemental Material).
Data analysis Both reaction time and ERP analyses were performed only on accurate trials. The statistical design was identical to that described in Experiment 1.

\section{Results and discussion}

Behavioral measures Analysis of RTs yielded a significant effect of Valence, $\mathrm{F}(2,28)=7.57, \mathrm{p}=.009, \eta_{\mathrm{p}}^{2}=.351$, indicating slower responses during viewing of emotional compared to neutral pictures, $\mathrm{F}_{\mathrm{s}}(1,14)>7.88, \mathrm{p}_{\mathrm{s}}<.014, \eta_{\mathrm{ps}}^{2}>.360$, and no difference between pleasant and unpleasant contents, $\mathrm{F}(1,14)=2.07, \mathrm{p}=.172$. Moreover, a significant main effect of Block was observed, $F(3,42)=9.47, p=.002, \eta_{p}^{2}=.403$, showing slower responses during block 1 compared to all other blocks, $\mathrm{F}_{\mathrm{s}}(1,14)>7.64, \mathrm{p}_{\mathrm{s}}<.05, \eta_{\mathrm{ps}}^{2}>.354$, and no difference among all other blocks, $\mathrm{F}_{\mathrm{s}}(1,14)<2.87, \mathrm{p}_{\mathrm{s}}>.112$. Finally, a significant Valence $\times$ Block interaction was observed, $\mathrm{F}(6,84)=3.17, \mathrm{p}=.012, \eta_{\mathrm{p}}^{2}=.185$. Following up on this interaction, emotional effects were investigated in each block. A significant effect of Valence was observed during block 1, $\mathrm{F}(2,28)=9.57, \mathrm{p}<.001, \eta_{\mathrm{p}}^{2}=.406$, with slower responses to emotional compared to neutral stimuli, $\mathrm{F}_{\mathrm{s}}(1,14)>9.68, \mathrm{p}_{\mathrm{s}}<.01$, $\eta_{\text {ps }}^{2}>$.409. In block 2 , the factor Valence was still statistically significant, $F(2,28)=4.66, p=.020, \eta_{p}^{2}=.250$, with slower responses to unpleasant compared to neutral stimuli, $\mathrm{F}(1,14)=6.01, \mathrm{p}=.028, \eta_{\mathrm{p}}^{2}=.300$. No valence effect was found in block 3. Crucially, affective modulation of RTs during the novel phase reached significance, Valence, $\mathrm{F}(2,28)=3.88, \mathrm{p}=.032, \eta_{\mathrm{p}}^{2}=.217$, showing slower responses to both pleasant and unpleasant compared to neutral stimuli, $\mathrm{F}_{\mathrm{s}}(1$, 14) $>6.79, p_{s}=.021, \eta_{p s}^{2}>.327$. Finally, difference scores (emotional minus neutral pictures) were calculated for block 4 (novel) and block 1, with the intent of comparing the emotional interference, and we found that the two blocks showed similar emotional effects, $\mathrm{F}(2,28)=2.19, \mathrm{p}=.130$.

Analysis of accuracy (see Table 1) failed to show any statistically significant main effect of Valence and Block, or Valence $\times$ Block interaction, $\mathrm{F}_{\mathrm{s}}<2.2, \mathrm{p}_{\mathrm{s}}>05$.

Late positive potential (LPP) $)^{2}$ As illustrated in Fig. 3, unpleasant and pleasant stimuli elicited larger positivity over centro-parietal sensors in the $400-800 \mathrm{~ms}$ time interval. This

\footnotetext{
${ }^{2}$ Further analyses were conducted to evaluate the effects of Block and Picture Valence on an early time interval (150-300 ms) over occipitotemporal regions (P2/EPN, early posterior negativity; Schupp, Junghöfer, Weike, \& Hamm, 2006) (cluster of sensors: TP7, TP8,T5, T6, P5, P6, PO7, PO8,PO5, PO6,PO3, PO4, O2, O1, POZ, OZ). Results indicated a main effect of Picture Valence on the early time interval (150-300 ms), $\mathrm{F}(2,28)=17.63, \mathrm{p}<.001, \eta_{\mathrm{ps}}^{2}=.558$, with pleasant contents eliciting less positivity compared to unpleasant and neutral ones, $(\operatorname{Fs}(1,14)>$ 25.42 , ps $<.001, \eta_{\mathrm{ps}}^{2}>.645, \mathrm{Fs}(1,14)>19.69, \eta_{\mathrm{ps}}^{2}<.001, \eta_{\mathrm{ps}}^{2}>.584$, respectively). No significant main effect of Block was observed, $\mathrm{F}=2.5$, $\mathrm{p}=.148$. Moreover, the interaction Valence $\times$ Block failed to reach significance, $\mathrm{F}(6,84)=1.09, \mathrm{p}=.372$
} 


\section{EXPERIMENT 2}
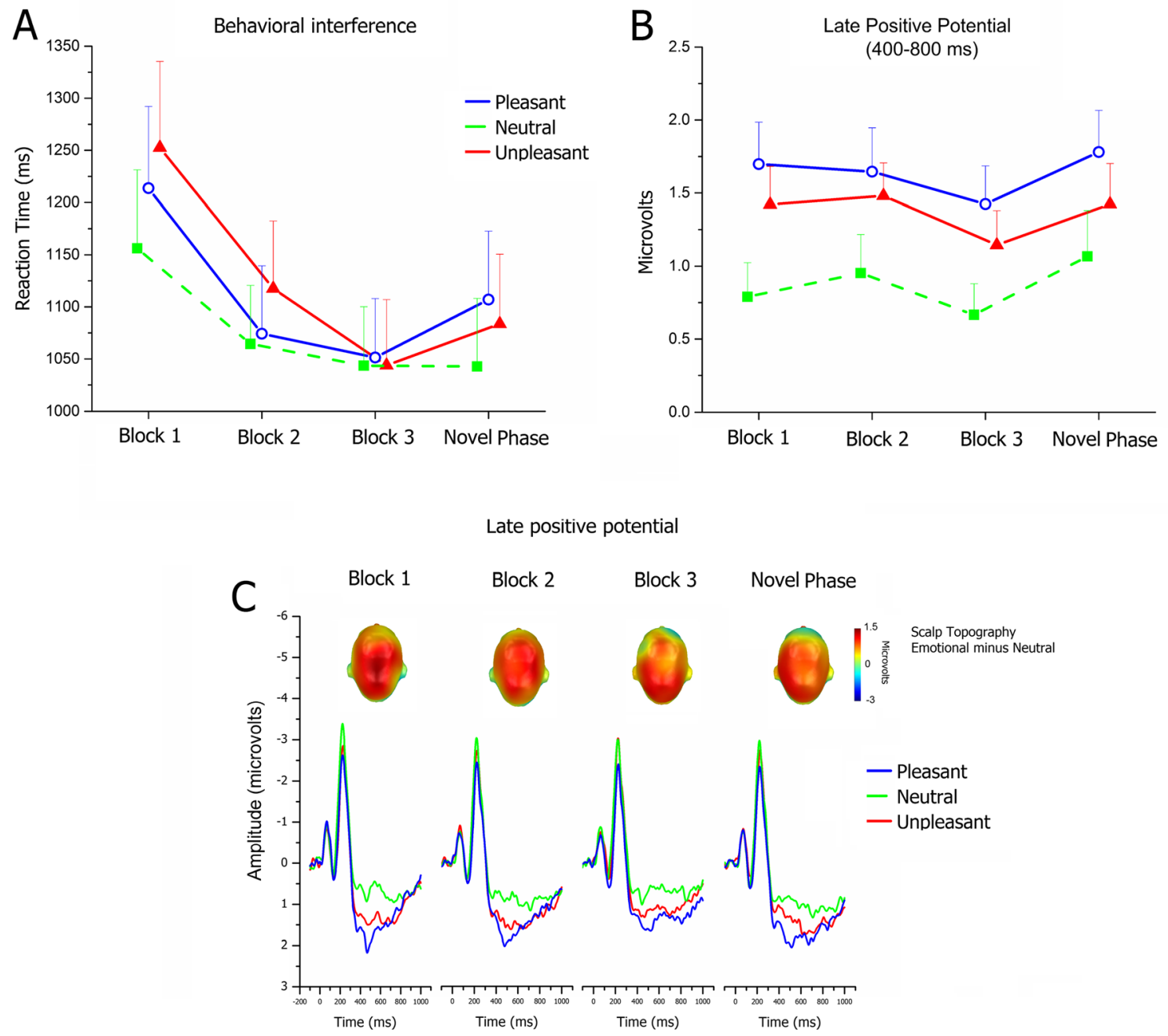

Fig. 3 Top: Response times (A) in the parity judgment task and LPP amplitude (B) across the three habituation blocks, and one novel block, plotted by distractor valence. Error bars represent one standard error of the mean. Bottom: Grand-averaged ERP waveforms (C) averaged over centroparietal sensors used for the analysis of the LPP for pleasant,

emotional modulation of the LPP was not affected by picture repetition and persisted across the habituation blocks.

The late positive potential was modulated by picture Valence, $\mathrm{F}(2,28)=13.43, \mathrm{p}<.001, \eta_{\mathrm{p}}^{2}=.490$, with pleasant and unpleasant contents eliciting a more pronounced late positive potential compared to neutral ones, $\mathrm{F}_{\mathrm{s}}(1,14)>17.22, \mathrm{p}_{\mathrm{s}}<.001$, $\eta_{\mathrm{ps}}^{2}>$.552. LPP amplitude for pleasant and unpleasant pictures did not differ, $\mathrm{F}(1,14)<1.65, \mathrm{p}=.216$. No significant main effect of Block was observed, $\mathrm{F}<2.2, \mathrm{p}>.05$. Moreover, the interaction Valence $\times$ Block failed to reach significance, $\mathrm{F}(6,78)$ $=.248, \mathrm{p}=.930$, indicating that affective modulation of the late positive potential did not vary across blocks. Consistently, the LPP amplitude continued to be enhanced during viewing of taskirrelevant emotional, compared to neutral pictures, regardless of repetition (in all blocks, $\mathrm{F}_{\mathrm{s}}(2,28)>4.33, \mathrm{p}_{\mathrm{s}}<.05, \eta_{\mathrm{ps}}^{2}>.223$ ).

neutral, and unpleasant pictures in the habituation phase and in the novel phase. The inset in each graph illustrates the scalp topography (400-800 ms) of the difference between emotional and neutral picture processing

Correlation between the LPP and Behavioral measures In order to directly assess the relationship between the centroparietal late positive potential (LPP) and the behavioral measures (RT and accuracy), we examined the correlations between these measures. Pearson's correlation coefficients were calculated between the LPP and RT and accuracy for each condition and participant. An additional analysis was carried out using the difference scores (emotional minus neutral) for each block. None of the correlations were significant $\left(\mathrm{r}_{\mathrm{s}}<.33\right.$, $\mathrm{p}_{\mathrm{s}}>.22$ ).

Similar to Experiment 1, the behavioral interference effect of emotional stimuli (pleasant and unpleasant) on the parity judgment task declined with stimulus repetition, and recovered completely when novel emotional pictures were presented. The affective modulation of the LPP, however, was not 
affected by stimulus repetition. Emotional stimuli continued to elicit a larger LPP than neutral pictures even after several repetitions of the same scene. Finally, and possibly because of more practice having taken place, the effect size of emotional interference in the first block was higher, and more resistant to stimulus repetition, in Experiment 2 than in Experiment 1 $\left(\eta_{p}^{2}=.41\right.$ vs. .15), consistent with previous findings that showed that as practice of the task increases interference from distracting information becomes stronger (Harris \& Pashler, 2004; Wilson, MacLeod, \& Muroi, 2008).

\section{General discussion}

To examine the processing of task-irrelevant emotional stimuli, we conducted two experiments that investigated the effects of stimulus repetition on behavioral interference and the late positive potential. Findings showed that the interference effect of emotional stimuli (pleasant and unpleasant) on the ongoing task declined with stimulus repetition, suggesting that the emotional content of stimuli that had been previously presented did not affect picture processing any longer. To better clarify this issue we recorded ERPs in Experiment 2, and we found that the affective modulation of the LPP was preserved despite picture repetition, even when emotional stimuli were distractors, and no behavioral emotional interference was observed. Therefore, altogether these findings indicate that emotional stimuli continue to engage motivational systems even when behavioral emotional interference on the primary task is inhibited. In both experiments, emotional interference recovered completely when novel pictures were presented, indicating that the decline of the emotional interference did not reflect a general habituation to task-irrelevant emotional stimuli but, rather, it was stimulus-specific.

Although RTs are often used to index attention to emotional stimuli (Calvo et al., 2015; Ferrari, Bruno, Chattat, \& Codispoti, 2016; Hartikainen et al., 2000; Padmala \& Pessoa, 2014; Weinberg \& Hajcak, 2011), they are executed after a sequence of processing stages following the actual attention allocation to the stimulus, and therefore several factors might affect behavioral interference. On the other hand, the LPP is a much more temporally proximal measure of the evaluative processes involved. Therefore, the LPP can reflect emotional engagement, even when attentional allocation does not impact the timing or choice in behavior, such as when participants may have learned to inhibit emotional interference. It is interesting to note that the dissociation between the affective modulation of the LPP and emotional interference reported in the present study is also consistent with the literature on behavioral deviance distraction (Parmentier, 2014). In studies examining the processing of physically deviant stimuli, increasing evidence indicates that ERPs (e.g., P3a) and behavioral measures of distraction are often dissociated, suggesting that behavioral distraction appears to be controlled by further, and presumably later, mechanisms (Parmentier, 2014; Schröger \& Wolff, 1998; Wetzel, Schröger, \& Widmann, 2013).

In several previous studies, the emotional modulation of the LPP and the behavioral interference were used, to some extent, interchangeably to examine the effects of emotion on attention (De Cesarei \& Codispoti, 2008; Erthal et al., 2005; Ihssen, et al., 2007; MacNamara \& Hajack, 2009; Padmala \& Pessoa, 2014; Schupp et., al., 2006), and it has also been suggested that these two indexes are correlated (Weinberg \& Hajcak, 2011). The present study indicates that, although the LPP and RTs are both modulated by motivational significance (emotional arousal) during the viewing of novel pictures, they are differentially modulated by stimulus repetition, suggesting that they do not reflect the same process. Additionally, in the present study, the behavioral interference did not correlate with the late positive potential. Indeed, a substantial body of work indicates that a range of physiological changes, which have been associated with the orienting response and emotional engagement, do not covary, suggesting that different components reflect different facets of the response to significant stimuli (Barry, 2006, 2009; Bradley, 2009). It has been shown that the affective modulation of skin conductance, pupil dilation and heart rate habituate rapidly with stimulus repetition and are reinstated only when new pictures are presented (Codispoti et al., 2006; Ferrari, De Cesarei, Mastria, Lugli, Baroni, Nicoletti, \& Codispoti, 2016), whereas the affective modulation of the LPP persists even after massive repetition. The decline of emotional interference of the present study is consistent with these findings, and suggests that orienting decreases with repetition because the stimulus is easily recognized (i.e., increased stimulus intake is not necessary), and it is clear that no adaptive action is necessary.

The affective modulation of orienting measures, and of behavioral interference is reinstated only when new stimuli are presented in the novel phase, suggesting that even when the affective modulation wanes during the habituation phase, the incoming stimuli are compared to the already established neural model (Cowan, 1988, 1995; Sokolov, 1963; Thompson, 2009). Otherwise it would not be possible to detect a discrepancy and therefore no reinstatement of the orienting responses would be observed when new stimuli were presented (Elliot \& Cowan, 2001). The reinstatement of affective modulation to new stimuli also suggests that the decline of emotional interference does not reflect a general inhibition of the processing of task-irrelevant emotional stimuli (or other unspecific learning factors such as filtering of distractor location; Kelley \& Yantis, 2009). Rather, this decrease in emotional interference seems to be due to a specific habituation to repeated stimuli, and not a generalization occurring in the entire semantic category to which the repeated pictures belong or to features that are shared between individual scenes, e.g., blood in unpleasant scenes (Gati \& BenShakar, 1990). 
Like the LPP, the affective modulation of the startle reflex is resistant to habituation (Bradley, Lang \& Cuthbert, 1993; Ferrari et al., 2011). Affective modulation of the blink reflex has been interpreted as reflecting motivational priming, in which a defensive reflex such as the startle is augmented when the ongoing motivational state is aversive (Lang, Bradley \& Cuthbert, 1990), and it has been shown that this modulation is not affected by stimulus repetition (Bradley et al., 1993; Ferrari et al., 2011).

However, the decline of emotional interference with stimulus repetition reported in the present study parallels the findings of another index of the orienting response: the Probe $\mathrm{P} 3$, that is the $\mathrm{P} 3$ component of the event-related potential elicited by an acoustic probe presented 1-2 $\mathrm{s}$ after picture onset. More specifically, the amplitude of this P3 is inhibited when pleasant or unpleasant pictures serve as the foreground stimuli, compared to neutral pictures (Bradley, Codispoti, \& Lang, 2006; Schupp, Cuthbert, Bradley, Birbaumer, \& Lang, 1997), indicating that less attention is available for probe processing when emotional, compared to neutral, pictures are being viewed. Recently we have shown that, unlike the LPP and the startle reflex, which continue to be modulated by emotional content following picture repetition, probe $\mathrm{P} 3$ amplitude is no longer different when viewing emotional, compared to neutral, pictures after multiple repetitions (Ferrari et al., 2011). This suggests that this component of the orienting response, like the behavioral emotional interference described here, reflects resource allocation, which is expected to diminish with stimulus repetition.

Although previous LPP studies have shown that the affective modulation of the LPP persists even after massive repetition, these studies examined the effect of stimulus repetition in free viewing tasks, where emotional stimuli were somehow attended, as participants were only required to look at the pictures (Codispoti et al., 2006; 2007). In the present study, we showed that picture repetition strongly reduced the behavioral emotional interference effect whereas affective cues continued to activate the neural circuits mediating appetitive or defensive motivation, as revealed by the affective modulation of the LPP, even when participants were actively engaged in an unrelated task, thus limiting the amount of resources that could be committed to the evaluation of affective, task-irrelevant stimuli.

Therefore these findings are not consistent with a mere attention-capture interpretation of the affective modulation of the LPP, but provide further support that this cortical modulation indexes motivational significance, defined as activation of cortico-limbic appetitive and defensive systems.

Our perceptual system serves the adaptive function of identifying an object or individual as a potential threat or reward, in order to act appropriately. We evaluate all incoming stimuli, even if they are repeated several times. One interpretation of our findings is that evaluation is mandatory in that it does not require the intention to make an evaluative judgment, and occurs even when emotional stimuli are task-irrelevant. Despite the number of repetitions, the new stimulus must be identified before it can be categorized as previously encountered, even when participants are actively engaged in an unrelated task. The evaluative system may orient the organism toward the stimulus (what is it?), and prepare the organism for action. While evaluative processes are mandatory, and continue to engage corticolimbic appetitive and defensive systems even after massive repetition, as suggested by the affective modulation of the LPP, attentional processes are not necessary after several repetitions of the same stimulus as indicated by the rapid decline of behavioral emotional interference. This is consistent with the idea that evaluative processing, and the engagement of the motivational systems, may occur independently of attentive processes, because the capacity to prevent limited resources from being allocated to repeated emotional stimuli increases the efficiency of the information processing system.

\section{References}

Anderson, B. A., Laurent, P. A., \& Yantis, S. (2011). Value-driven attentional capture. Proceedings of the National Academy of Sciences of the United States of America, 108(25), 10367-10371. doi:10.1073 /pnas. 1104047108

Barry, R. J. (2006). Promise versus reality in relation to the unitary orienting reflex: A case study examining the role of theory in psychophysiology. International Journal of Psychophysiology, 62, 353-366. doi:10.1016/j.ijpsycho.2006.01.004

Barry, R. J. (2009). Habituation of the orienting reflex and the development of preliminary process theory. Neurobiology of Learning and Memory, 92, 235-242. doi:10.1016/j.nlm.2008.07.007

Bayer, M., \& Schacht, A. (2014). Event-related brain responses to emotional words, pictures and faces - A cross-domain comparison. Frontiers in Psychology, 5, 1106. doi:10.3389/fpsyg.2014.01106

Bayer, M., Sommer, W., \& Schacht, A. (2011). Emotional Words Impact the Mind but not the Body: Evidence from Pupillary Responses. Psychophysiology, 48, 1553-1561. doi:10.1111/j.14698986.2011.01219.x

Bradley, M. M. (2009). Natural selective attention: Orienting and emotion. Psychophysiology, 46, 1-11. doi:10.1111/j.14698986.2008.00702.x

Bradley, M. M., Codispoti, M., \& Lang, P. J. (2006). A multi-process account of startle modulation during affective perception. Psychophysiology, 43, 486-497. doi:10.1111/j.14698986.2006.00412.x

Bradley, M. M., Cuthbert, B. N., \& Lang, P. J. (1999). Affect and the startle reflex. In Dawson, M. E., Schell, A. M., Bohmelt, A. H., Eds. Startle modification: Implications for neuroscience, cognitive science, and clinical science. Cambridge University Press. doi: 10.1017/CBO9780511665523.010

Bradley, M. M., Hamby, S., Löw, A., \& Lang, P. J. (2007). Brain potentials in perception: Picture complexity and emotional arousal. Psychophysiology, 44, 364-373. doi:10.1111/j.14698986.2007.00520.x

Bradley, M. M., Lang, P. J., \& Cuthbert, B. N. (1993). Emotion, novelty, and the startle reflex: Habituation in humans. Behavioral Neuroscience, 107, 970-980. doi:10.1037/0735-7044.107.6.970

Bradley, B. P., Mogg, K., Falla, S. J., \& Hamilton, L. R. (1998). Attentional bias for threatening facial expressions in anxiety: 
Manipulation of stimulus duration. Cognition and Emotion, 12, 737-753. doi:10.1080/026999398379411

Calvo, M. G., Gutiérrez-García, A., \& Del Líbano, M. (2015). Sensitivity to emotional scene content outside the focus of attention. Acta Psychologica, 161, 36-44. doi:10.1016/j.actpsy.2015.08.002

Codispoti, M., De Cesarei, A., \& Ferrari, V. (2012). The influence of color on emotional perception of natural scenes. Psychophysiology, 49, 11-16. doi:10.1111/j.1469-8986.2011.01284.x

Codispoti, M., Ferrari, V., \& Bradley, M. M. (2006). Repetitive picture processing: Autonomic and cortical correlates. Brain Research, 1068, 213-220. doi:10.1016/j.brainres.2005.11.009

Codispoti, M., Ferrari, V., \& Bradley, M. M. (2007). Repetition and event-related potentials: Distinguishing early and late processes in affective picture perception. Journal of Cognitive Neuroscience., 19, 577-586. doi:10.1162/jocn.2007.19.4.577

Codispoti, M., Mazzetti, M., \& Bradley, M. M. (2009). Unmasking emotion: Exposure duration and emotional engagement. Psychophysiology, 46, 731-738.

Cowan, N. (1988). Evolving conceptions of memory storage, selective attention, and their mutual constraints within the human information processing system. Psychological Bulletin, 104, 163-191. doi:10.1037/0033-2909.104.2.163

Cowan, N. (1995). Attention and memory: An integrated framework. New York: Oxford University Press.

Cuthbert, B. N., Schupp, H. T., Bradley, M. M., Birbaumer, N., \& Lang, P. J. (2000). Brain potentials in affective picture processing: Covariation with autonomic arousal and affective report. Biological Psychology, 52, 95-111. doi:10.1016/S0301-0511(99 )00044-7

De Cesarei, A., \& Codispoti, M. (2006). When does size not matter? Effects of stimulus size on affective modulation. Psychophysiology, 43, 207215. doi:10.1111/j.1469-8986.2006.00392.x

De Cesarei, A., \& Codispoti, M. (2008). Fuzzy picture processing: Effects of size reduction and blurring on emotional processing. Emotion, 8, 352-363. doi:10.1037/1528-3542.8.3.352

De Cesarei, A., \& Codispoti, M. (2011). Scene identification and emotional response: Which spatial frequencies are critical? Journal of Neuroscience, 31(47), 17052-17057. doi:10.1523/JNEUROSCI.374511.2011

De Houwer, J., \& Hermans, D. (1994). Differences in the affective processing of words and pictures. Cognition and Emotion, 8, 1-20. doi: $10.1080 / 02699939408408925$

Elliott, E. M., \& Cowan, N. (2001). Habituation to auditory distractors in a cross-modal, color-word interference task. Journal of Experimental Psychology: Learning, memory and Cognition, 27, 654-667. doi:10.1037/0278-7393.27.3.654

Erthal, F. S., de Oliveira, L., Mocaiber, I., Pereira, M. G., MachadoPinheiro, W., Volchan, E., \& Pessoa, L. (2005). Load-dependent modulation of affective picture processing. Cognitive Affective and Behavioral Neuroscience, 5, 388-395. doi:10.3758/CABN.5.4.388

Ferrari, V., Bradley, M. M., Codispoti, M., \& Lang, P. J. (2011). Repetitive exposures: Brain and reflex measures of emotion and attention. Psychophysiology, 48, 515-522. doi:10.1111/j.14698986.2010.01083.x

Ferrari, V., Bruno, N., Chattat, R., \& Codispoti, M. (2016). Evaluative ratings and attention across the life span: Emotional arousal and gender. Cognition and Emotion,, 1-12. doi:10.1080 /02699931.2016.1140020

Ferrari, V., De Cesarei, A., Mastria, S., Lugli, L., Baroni, G., Nicoletti, R., \& Codispoti, M. (2016). Novelty and emotion: Pupillary and cortical responses during viewing of natural scenes. Biological Psychology, 113, 75-82. doi:10.1016/j.biopsycho.2015.11.008

Ferrari, V., Mastria, S., \& Bruno, N. (2014). Crossmodal interactions during affective picture processing. PLoS One, 9, e89858. doi:10.1371/journal.pone. 0089858
Gati, I., \& Ben-Shakhar, G. (1990). Novelty and significance in orientation and habituation: A feature-matching approach. Journal of Experimental Psychology: General, 119, 251-263. doi:10.1037 /0096-3445.119.3.251

Gottlieb, J. (2012). Attention, learning, and the value of information. Neuron, 76(2), 281-295. doi:10.1016/j.neuron.2012.09.034

Gratton, G., Coles, M. G., \& Donchin, E. (1983). A new method for offline removal of ocular artifact. Electroencephalography and Clinical Neurophysiology, 55, 468-484. doi:10.1016/0013-4694 (83) $90135-9$

Gronau, N., Cohen, A., \& Ben-Shakhar, G. (2009). Distractor interference in focused attention tasks is not mediated by attention capture. The Quarterly Journal of Experimental Psychology, 62(9), 16851695. doi: $10.1080 / 17470210902811223$

Harris, C. R., \& Pashler, H. (2004). Attention and the processing of emotional words and names: Not so special after all. Psychological Science, 15, 171-178. doi:10.1111/j.09567976.2004.01503005.x

Hartikainen, K. M., Ogawa, K. H., \& Knight, R. T. (2000). Transient interference of right hemispheric function due to automatic emotional processing. Neuropsychologia, 38, 1576-1580. doi:10.1016 /S0028-3932(00)00072-5

Hickey, C., Chelazzi, L., \& Theeuwes, J. (2010). Reward changes salience in human vision via the anterior cingulate. Journal of Neuroscience, 30(33), 11096-11103. doi:10.1523/JNEUROSCI.1026-10.2010

Hinojosa, J. A., Carretié, L., Valcárcel, M. A., Méndez-Bértolo, C., \& Pozo, M. A. (2009). Electrophysiological differences in the processing of affective information in words and pictures. Cognitive Affective and Behavioral Neuroscience, 9, 173-189. doi:10.3758 /CABN.9.2.173

Ihssen, N., Heim, S., \& Keil, A. (2007). The costs of emotional attention: Affective processing inhibits subsequent lexico-semantic analysis. Journal of Cognitive Neuroscience, 19, 1932-1949. doi:10.1162 jocn.2007.19.12.1932

Johnston, V. S., Miller, D. R., \& Burleson, M. H. (1986). Multiple P3s to emotional stimuli and their theoretical significance. Psychophysiology, 23, 684-694. doi:10.1111/j.1469-8986.1986. tb00694.x

Junghöfer, M., Elbert, T., Tucker, D. M., \& Rockstroh, B. (2000). Statistical control of artifacts in dense array EEG/MEG studies. Psychophysiology, 37, 523-532. doi:10.1111/1469-8986.3740523

Kelley, T., \& Yantis, S. (2009). Learning to attend: Effects of practice on information selection. Journal of Vision, 9, 1-18. doi:10.1167 /9.7.16

Lang, P., \& Bradley, M. M. (2010). Emotion and the motivational brain. Biological Psychology, 84, 437-450. doi:10.1016/j. biopsycho.2009.10.007

Lang, P. J., Bradley, M. M., \& Cuthbert, B. N. (1990). Emotion, attention, and the startle reflex. Psychological Review, 97, 377-395. doi: 10.1037/0033-295X.97.3.377

Lang, P. J., Bradley, M. M., \& Cuthbert, B. N. (1997). Motivated attention: Affect, activation, and action. In P. J. Lang, R. F. Simons, \& M. Balaban (Eds.), Attention and orienting (pp. 97-135). Mahwah: Erlbaum.

Lang, P. J., Bradley, M. M., \& Cuthbert, B. N. (2008). International affective picture system (IAPS): Affective ratings of pictures and instruction manual. Technical Report A-8. Gainesville: University of Florida.

LeDoux, J. (2012). Rethinking the emotional brain. Neuron, 73(5), 1052. doi:10.1016/j.neuron.2012.02.018

MacNamara, A., \& Hajcak, G. (2009). Anxiety and spatial attention moderate the electrocortical response to aversive pictures. Neuropsychologia, 47, 2975-2980. doi:10.1016/j.neuropsychologia.2009.06.026

Mitchell, D., Richell, R., Leonard, A., \& Blair, R. (2006). Emotion at the expense of cognition: Psychopathic individuals outperform controls 
on an operant response task. Journal of Abnormal Psychology, 115, 559. doi:10.1037/0021-843X.115.3.559

Most, S. B., Smith, S. D., Cooter, A. B., Levy, B. N., \& Zald, D. H. (2007). The naked truth: Positive, arousing distractors impair rapid target perception. Cognition and Emotion, 21, 961-981. doi:10.1080/02699930600959340

Öhman, A. (1992). Orientingand attention: Preferred preattentive processing of potentially phobic stimuli. In B. A. Campbell, H. Hayne, \& R. Richardson (Eds.), Attention and Information Processing in Infants and Adults (pp. 263-295). Hillsdale: Erlbaum.

Padmala, S., \& Pessoa, L. (2014). Motivation versus aversive processing during perception. Emotion, 14, 450-454. doi:10.1037/a0036112

Parmentier, F. B. R. (2014). The cognitive determinants of behavioral distraction by deviant auditory stimuli: A review. Psychological Research, 78, 321-338. doi:10.1007/s00426-013-0534-4

Peyk, P., De Cesarei, A., \& Junghöfer, M. (2011). ElectroMagneto EncephaloGraphy Software (EMEGS): overview and integration with other EEG/MEG toolboxes. Computational Intelligence and Neuroscience, 861705. doi: 10.1155/2011/861705

Pourtois, G., Schettino, A., \& Vuilleumier, P. (2013). Brain mechanisms for emotional influences on perception and attention: What is magic and what is not. Biological Psychology, 92, 492-512. doi:10.1016/j. biopsycho.2012.02.007

Qu, Z., Hillyard, S. A., \& Ding, Y. (2016). Perceptual learning induces persistent attentional capture by nonsalient shapes. Cerebral Cortex. doi:10.1093/cercor/bhv342

Raymond, J. E., \& O'Brien, J. L. (2009). Selective visual attention and motivation: The consequences of value learning in an attentional blink task. Psychological Science, 20(8), 981-988. doi:10.1111 jj.1467-9280.2009.02391.x

Schimmack, U. (2005). Attentional Interference Effects of Emotional Pictures: Threat, Negativity, or Arousal? Emotion, 5, 55-66. doi:10.1037/1528-3542.5.1.55

Schneider, W., Eschman, A., \& Zuccolotto, A. (2002). E-prime User's Guide. Pittsbourgh: Psychology Software Tools Inc.

Schröger, E., \& Wolff, C. (1998). Behavioral and electrophysiological effects of task-irrelevant sound change: A new distraction paradigm.
Cognitive Brain Research, 7, 71-87. doi:10.1016/s0926-6410(98 00013-5

Schupp, H. T., Cuthbert, B. N., Bradley, M. M., Birbaumer, N., \& Lang, P. J. (1997). Probe P3 and blinks: Two measures of affective startle modulation. Psychophysiology, 34, 1-6. doi:10.1111/j.14698986.1997.tb02409.x

Schupp, H. T., Flaisch, T., Stockburger, J., \& Junghöfer, M. (2006). Emotion and attention: Event-related brain potential studies. Progress Brain Research, 156, 31-51. doi:10.1016/S0079-6123 (06)56002-9

Sokolov, E. N. (1963). Perception and the conditioned reflex. New York: Macmillan.

Thompson, R. F. (2009). Habituation: A history. Neurobiology of Learning and Memory, 92(2), 127-134. doi:10.1016/j. nlm.2008.07.011

Turatto, M., \& Pascucci, D. (2016). Short-term and long-term plasticity in the visual-attention system: Evidence from habituation of attentional capture. Neurobiology of Learning and Memory, 130, 159-169. doi:10.1016/j.nlm.2016.02.010

Vuilleumier, P., Armony, J. L., Driver, J., \& Dolan, R. J. (2001). Effects of attention and emotion on face processing in the human brain: An event-related fMRI study. Neuron, 30, 829-841. doi:10.1016 /S0896-6273(01)00328-2

Weinberg, A., \& Hajcak, G. (2011). The late positive potential predicts subsequent interference with target processing. Journal of Cognitive Neuroscience, 23, 2994-3007. doi:10.1162/jocn.2011.21630

Wetzel, N., Schröger, E., \& Widmann, A. (2013). The dissociation between the P3a event-related potential and behavioral distraction. Psychophysiology, 50, 920-930. doi:10.1111/psyp.12072

Wilson, D. E., MacLeod, C. M., \& Muroi, M. (2008). Practice in visual search produces decreased capacity demands but increased distraction. Perception \& Psychophysics, 70, 1130-1137. doi:10.3758 /PP.70.6.1130

Wolford, G., \& Morrison, F. (1980). Processing of unattended visual information. Memory \& Cognition, 8, 521-527. doi:10.3758 /BF03213771 